\title{
Dynamics Analysis of Game and Chaotic Control in the Chinese Fixed Broadband Telecom Market
}

\author{
Jia Liu, ${ }^{1,2}$ Guoliang Liu, ${ }^{1} \mathrm{Na} \mathrm{Li,}{ }^{1}$ and Hongliang $\mathrm{Xu}^{3}$ \\ ${ }^{1}$ Management School, Jilin University, Changchun 130025, China \\ ${ }^{2}$ Heilongjiang Admission and Examinations Committee Office, Harbin 150090, China \\ ${ }^{3}$ School of Life Science and Technology, Mudanjiang Normal University, Mudanjiang 157011, China \\ Correspondence should be addressed to Guoliang Liu; glliug@jlu.edu.cn
}

Received 8 October 2013; Revised 6 April 2014; Accepted 17 April 2014; Published 19 May 2014

Academic Editor: Manuel De la Sen

Copyright (c) 2014 Jia Liu et al. This is an open access article distributed under the Creative Commons Attribution License, which permits unrestricted use, distribution, and reproduction in any medium, provided the original work is properly cited.

\begin{abstract}
This paper considers a dynamic duopoly Cournot model based on nonlinear cost functions. The model with heterogeneous players and the spillover effect is applied to the Chinese fixed broadband telecom market. We have studied its dynamic game process. The existence and stability of the Nash equilibrium of the system have been discussed. Simulations are used to show the complex dynamical behaviors of the system. The results illustrate that altering the relevant parameters of system can affect the stability of the Nash equilibrium point and cause chaos to occur. With the use of the delay feedback control method, the chaotic behavior of the model has been stabilized at the Nash equilibrium point. The analysis and results will be of great importance for the Chinese fixed broadband telecom market.
\end{abstract}

\section{Introduction}

The classical oligopoly model was proposed by the French economist Augustine Cournot. In recent years, on the basis of classic Cournot and Bertrand models, many factors such as bounded rationality, nonlinear cost, the spillover effect, delayed decision-making, and incomplete information have been introduced into game models [1-7]. In these studies, researchers have presented improved dynamical oligopoly game models and discussed the complicated dynamics of the whole game process.

To control the chaos in the game model, many successful applications of chaos control methods are used in economic systems $[8,9]$. The OGY method has been used by Agiza [10] to control the chaos in economic systems, which requires exact system information before implementation. The straight-line stabilization method has been applied by Yao et al. [11] to control chaos in an investment model. A modified straight-line stabilization method has been used by $\mathrm{Du}$ et al. [12] in an output duopoly competing evolution model. The time-delayed feedback control method has been used by Holyst and Urbanowicz [13] to control chaos in an economical model. In contrast, the delayed feedback control method can be easily applied without requiring any system information, though it has some other drawbacks.

Fixed broadband is a part of the Chinese telecom market and is a typical oligopoly industry. When the China telecom industry was restructured in 2008, three pillars in the China telecom market have emerged: the China Telecommunications Corporation (China Telecom), the China United Telecommunications (China Unicom), and the China Mobile Communications Group (China Mobile). The fixed broadband business is mainly managed by China Unicom and China Telecom. They control most of the Chinese fixed broadband market, in which a duopoly situation has formed. The Bertrand model was applied to the Chinese 3G telecom market in a literature [14]. To study the competitive process of the Chinese fixed broadband market, this paper considers a dynamical Cournot model, which is provided with heterogeneous players, nonlinear cost functions, and the spillover effect. We analyze the complex dynamic behaviors of the game model and apply a delay feedback control method to control the chaos of the system. Then, the chaotic 
phenomena of the fixed broadband market are delayed and even eliminated gradually.

\section{The Model and Its Stability Analysis}

2.1. The Model. In the duopoly fixed broadband market, $q_{i}(t)$ denotes the quantity supplied by telecom operator $i(i=1,2)$ during period $t$. In addition, there is an inverse demand function that has the form $P=P(Q)=a-b Q$, where $Q(t)=q_{1}(t)+q_{2}(t)$ is the total quantity supplied and $a$ and $b$ are positive constants. Thus, the nonlinear cost function $C_{i}\left(q_{i}\right)=\left(c_{i} q_{i}^{2}+d_{i} q_{i}+e_{i}\right) /\left(1+r_{i j} q_{j}\right)$ is defined, where $c_{i}, d_{i}$, and $e_{i}$ are positive constants concerning telecom technology. The parameter $r_{i j}$ is the spillover effect and $r_{i j} \geq 0$ characterizes the positive cost externality in the cost of operator $i$ related to the presence of operator $j$ [15]. By using the equations above, the profit resulting of operators $i$ is given by

$$
\Pi_{i}=q_{i}(a-b Q)-\left(\frac{c_{i} q_{i}^{2}+d_{i} q_{i}+e_{i}}{1+r_{i j} q_{j}}\right), \quad i=1,2 .
$$

Then, the marginal profit of operator $i$ is given by

$$
\begin{array}{r}
\Phi_{i}=\frac{\partial \Pi_{i}}{\partial q_{i}}=\left(a-\frac{d_{i}}{1+r_{i j} q_{j}}\right)-2\left(b+\frac{c_{i}}{1+r_{i j} q_{j}}\right) q_{i}-b q_{j}, \\
i=1,2, \quad i \neq j .
\end{array}
$$

This optimization problem has a unique solution in the form

$$
q_{i}^{*}=\frac{1}{2\left(b+\left(c_{i} /\left(1+r_{i j} q_{j}\right)\right)\right)}\left(\left(a-\frac{d_{i}}{1+r_{i j} q_{j}}\right)-b q_{j}\right) .
$$
(3).

If the operator is naive, he decides his output according to

If the operator is a bounded rational one, he does not have a complete knowledge of the demand function of the market and builds his output decision on the basis of the expected marginal profit. If the marginal profit is positive (negative), he increases (decreases) its production $q_{i}$ at the next period output. Then, the dynamical equation of player $i$ has the form below [16]

$$
q_{i}(t+1)=q_{i}(t)+\alpha q_{i}(t) \Phi_{i}(t)
$$

where $\alpha$ is a positive parameter and represents the relative speed of adjustment of the operator. In this paper, we consider that the first operator is a bounded rational one and that the second is a naive one. By inserting (2) into (4), we can obtain a dynamical game model with heterogeneous players in the form

$$
\begin{aligned}
& q_{1}(t+1) \\
& =q_{1}(t)+\alpha q_{1}(t) \\
& \quad \times\left(\left(a-\frac{d_{1}}{1+r_{12} q_{2}}\right)-2\left(b+\frac{c_{1}}{1+r_{12} q_{2}}\right) q_{1}(t)-b q_{2}(t)\right) \\
& q_{2}(t+1)=\frac{1}{2\left(b+\left(c_{2} /\left(1+r_{21} q_{1}\right)\right)\right)} \\
& \quad \times\left(\left(a-\frac{d_{2}}{1+r_{21} q_{1}}\right)-b q_{1}(t)\right) .
\end{aligned}
$$

2.2. The Existence and Stability of the Equilibrium Point. To study the local stability of equilibrium points, the eigenvalues of the Jacobian matrix of system (5) have to be considered. The Jacobian matrix at the point $\left(q_{1}, q_{2}\right)$ has the form

$$
J=\left[\begin{array}{ll}
J_{11} & J_{12} \\
J_{21} & J_{22}
\end{array}\right]
$$

where

$$
\begin{gathered}
J_{11}=1+\alpha\left(a-\frac{d_{1}}{1+r_{12} q_{2}}-\left(4 b+4 \frac{c_{1}}{1+r_{12} q_{2}}\right) q_{1}-b q_{2}\right), \\
J_{12}=\alpha q_{1}\left(\frac{d_{1} r_{12}+2 c_{1} r_{12} q_{1}}{\left(1+r_{12} q_{2}\right)^{2}}-b\right), \\
J_{21}=\frac{\left(d_{2} r_{21} /\left(1+r_{21} q_{1}\right)^{2}\right)-b}{2 b+2\left(c_{2} /\left(1+r_{21} q_{1}\right)\right)} \\
+\frac{\left(2 a-2\left(d_{2} /\left(1+r_{21} q_{1}\right)\right)-2 b q_{1}\right) c_{2} r_{21}}{\left(2 b+2\left(c_{2} /\left(1+r_{21} q_{1}\right)\right)\right)^{2}\left(1+r_{21} q_{1}\right)^{2}} \\
J_{22}=0 .
\end{gathered}
$$

Then local stability of the Nash equilibrium point $E^{*}$ is discussed as follows.

Proposition 1. The Nash equilibrium $E^{*}$ of system (5) is stable provided that

$$
\begin{aligned}
\alpha^{*}<2\left(q_{1}^{*}\right. & \left(\frac{d_{1} r_{12}+2 c_{1} r_{12} q_{1}^{*}}{\left(1+r_{12} q_{2}^{*}\right)^{2}}-b\right) \\
\times & \left(\frac{\left(d_{2} r_{21} /\left(1+r_{21} q_{1}^{*}\right)^{2}\right)-b}{2 b+2\left(c_{2} /\left(1+r_{21} q_{1}^{*}\right)\right)}\right. \\
& \left.+\frac{\left(2 a-2\left(d_{2} /\left(1+r_{21} q_{1}^{*}\right)\right)-2 b q_{1}^{*}\right) c_{2} r_{21}}{\left(2 b+2\left(c_{2} /\left(1+r_{21} q_{1}^{*}\right)\right)\right)^{2}\left(1+r_{21} q_{1}^{*}\right)^{2}}\right)^{-1} \\
& \left.-\left(a-\frac{d_{1}}{1+r_{12} q_{2}^{*}}-\left(4 b+4 \frac{c_{1}}{1+r_{12} q_{2}^{*}}\right) q_{1}^{*}-b q_{2}^{*}\right)\right)^{-}
\end{aligned}
$$


The Jacobian matrix of system at $E^{*}$ takes the form

$$
J\left(E^{*}\right)=\left[\begin{array}{ll}
J_{11}^{*} & J_{12}^{*} \\
J_{21}^{*} & J_{22}^{*}
\end{array}\right] .
$$

Its characteristic equation is $F(\lambda)=\lambda^{2}-\operatorname{Tr} \lambda+$ Det $=0$, where $\operatorname{Tr}$ is the trace and Det is the determinant of matrix $J\left(E^{*}\right)$, which are given by

$$
\begin{aligned}
\operatorname{Tr}=1+ & \alpha\left(a-\frac{d_{1}}{1+r_{12} q_{2}^{*}}-\left(4 b+4 \frac{c_{1}}{1+r_{12} q_{2}^{*}}\right) q_{1}^{*}-b q_{2}^{*}\right) \\
\text { Det }= & -\alpha q_{1}^{*}\left(\frac{d_{1} r_{12}+2 c_{1} r_{12} q_{1}^{*}}{\left(1+r_{12} q_{2}^{*}\right)^{2}}-b\right) \\
& \times\left(\frac{\left(d_{2} r_{21} /\left(1+r_{21} q_{1}^{*}\right)^{2}\right)-b}{2 b+2\left(c_{2} /\left(1+r_{21} q_{1}^{*}\right)\right)}\right. \\
& \left.+\frac{\left(2 a-2\left(d_{2} /\left(1+r_{21} q_{1}^{*}\right)\right)-2 b q_{1}^{*}\right) c_{2} r_{21}}{\left(2 b+2\left(c_{2} /\left(1+r_{21} q_{1}^{*}\right)\right)\right)^{2}\left(1+r_{21} q_{1}^{*}\right)^{2}}\right) .
\end{aligned}
$$

We can get $\operatorname{Tr}^{2}-4$ Det $>0$. Thus, the eigenvalues of the Nash equilibrium are real. According to the Jury condition, the necessary and sufficient condition for the local stability of Nash equilibrium is obtained:

$$
\begin{gathered}
1-\operatorname{Tr}+\text { Det }>0 \\
1+\operatorname{Tr}+\text { Det }>0 \\
\text { Det }-1<0
\end{gathered}
$$

We have the stable region of Nash equilibrium point $E^{*}$ which is $\left[0, \alpha^{*}\right]$, where

$$
\begin{aligned}
\alpha^{*}=2\left(q_{1}^{*}\right. & \left(\frac{d_{1} r_{12}+2 c_{1} r_{12} q_{1}^{*}}{\left(1+r_{12} q_{2}^{*}\right)^{2}}-b\right) \\
& \times\left(\frac{\left(d_{2} r_{21} /\left(1+r_{21} q_{1}^{*}\right)^{2}\right)-b}{2 b+2\left(c_{2} /\left(1+r_{21} q_{1}^{*}\right)\right)}\right. \\
& \left.+\frac{\left(2 a-2\left(d_{2} /\left(1+r_{21} q_{1}^{*}\right)\right)-2 b q_{1}^{*}\right) c_{2} r_{21}}{\left(2 b+2\left(c_{2} /\left(1+r_{21} q_{1}^{*}\right)\right)\right)^{2}\left(1+r_{21} q_{1}^{*}\right)^{2}}\right)^{-1} \\
& \left.-\left(a-\frac{d_{1}}{1+r_{12} q_{2}^{*}}-\left(4 b+4 \frac{c_{1}}{1+r_{12} q_{2}^{*}}\right) q_{1}^{*}-b q_{2}^{*}\right)\right)^{-1} .
\end{aligned}
$$

We can obtain the information on the effects of parameter $\alpha$ on the stability of the Nash equilibrium $E^{*}=\left(q_{1}^{*}, q_{2}^{*}\right)$ with other parameters held fixed. The Nash equilibrium point $E^{*}=\left(q_{1}^{*}, q_{2}^{*}\right)$ is the stable point when parameter $\alpha<\alpha^{*}$. If the adjustment speed of the operator with bounded rationality is adjusted in $\left[0, \alpha^{*}\right]$, the production of the two operators will tend towards the Nash equilibrium point $E^{*}$ after rounds of games. The first operator adjusts its production at period $t+1$ according to the marginal profit at period $t$ and the second operator adjusts production according to the production of the first operator in the previous period. To obtain more profits, the first operator will increase their adjustment speed $\alpha$. Once the speed is too high, the equilibrium point $E^{*}$ will be out of the stable region when $\alpha=\alpha^{*}$, and perioddoubling bifurcations will appear. As parameter $\alpha$ increases continuously, the equilibrium point $\left(q_{1}^{*}, q_{2}^{*}\right)$ is unstable, and the system will fall into chaos when $\alpha>\alpha^{*}$.

When quantity supplied in the market reaches Nash equilibrium point $\left(q_{1}^{*}, q_{2}^{*}\right)$, the whole fixed broadband market is in the stable state. All the operators in the fixed broadband market are compared orderly. Economic meaning of the stable region is that whatever initial quantity supplied is chosen by two fixed broadband companies in local stable region, they will eventually achieve Nash equilibrium quantity $\left(q_{1}^{*}, q_{2}^{*}\right)$ after finite games. It is noticeable that the two fixed broadband companies cannot reach the Nash equilibrium point $\left(q_{1}^{*}, q_{2}^{*}\right)$ at once. They have to reach the equilibrium $\left(q_{1}^{*}, q_{2}^{*}\right)$ after rounds of games. In order to get more quantities and more profits, the fixed broadband operators will accelerate the adjustment speed of the quantity. But once one operator is adjusting quantity speed too fast and pushing $\alpha$ out of the stable region, the fixed broadband market tends to become unstable and fall into chaos. But the value of adjustment speed $\alpha$ will not change the Nash equilibrium quantity $\left(q_{1}^{*}, q_{2}^{*}\right)$.

\section{Chaos Analysis and Numerical Simulations of the System}

To study local stability of the Nash equilibrium point, it is convenient to consider the following set of parameters: $a=8$, $b=0.5, c_{1}=2, c_{2}=3, d_{1}=d_{2}=2, e_{1}=e_{2}=1, r_{12}=0.5$, and $r_{21}=1$. Inserting the values of parameters into (5),

$$
\begin{aligned}
& q_{1}(t+1) \\
& =q_{1}(t)+\alpha q_{1}(t) \\
& \quad \times\left(8-\frac{2}{1+0.5 q_{2}(t)}\right. \\
& \left.\quad-\left(1+\frac{4}{1+0.5 q_{2}(t)}\right) q_{1}(t)-0.5 q_{2}(t)\right) \\
& q_{2}(t+1) \quad \frac{1}{1+\left(6 /\left(1+q_{1}(t)\right)\right)}\left(8-\frac{2}{\left(1+q_{1}(t)\right)}-0.5 q_{1}(t)\right) .
\end{aligned}
$$

Nash equilibrium point $E^{*}$ has been obtained: $E^{*}=\left(q_{1}^{*}, q_{2}^{*}\right)=$ (2.030, 2.122).

The stability of the Nash equilibrium point $E^{*}$ has been analyzed by inserting the values of parameters into (11). It is obvious that $1-\operatorname{Tr}+$ Det $>0$ and Det $-1<0$. By $1+\operatorname{Tr}+$ Det $>$ 0 , parameter $\alpha$ has been obtained, $\alpha<0.3082$. It demonstrates that point $E^{*}$ is locally stable when parameter $\alpha<$ 0.3082 according to Figures 1 and 2 . 


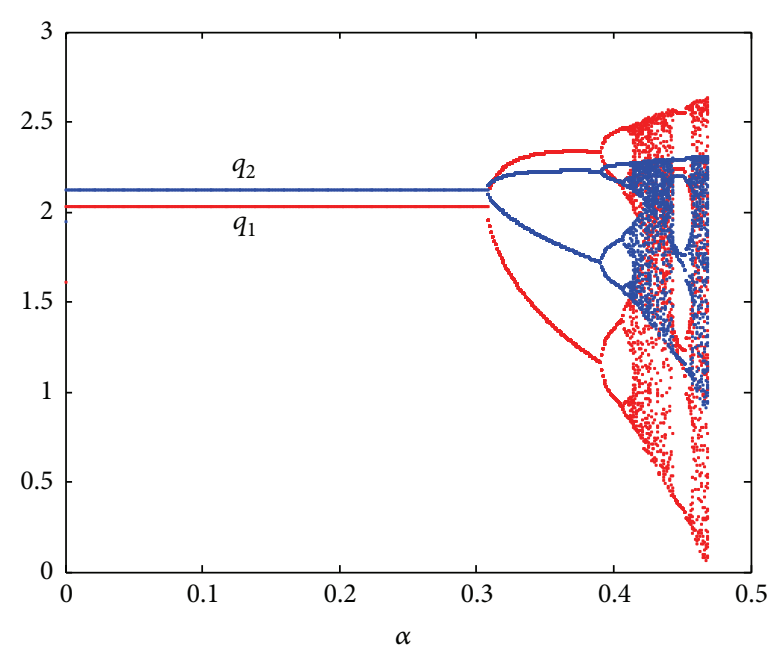

FIGURE 1: The bifurcation diagram of the system (13) with respect to the parameter $\alpha$.

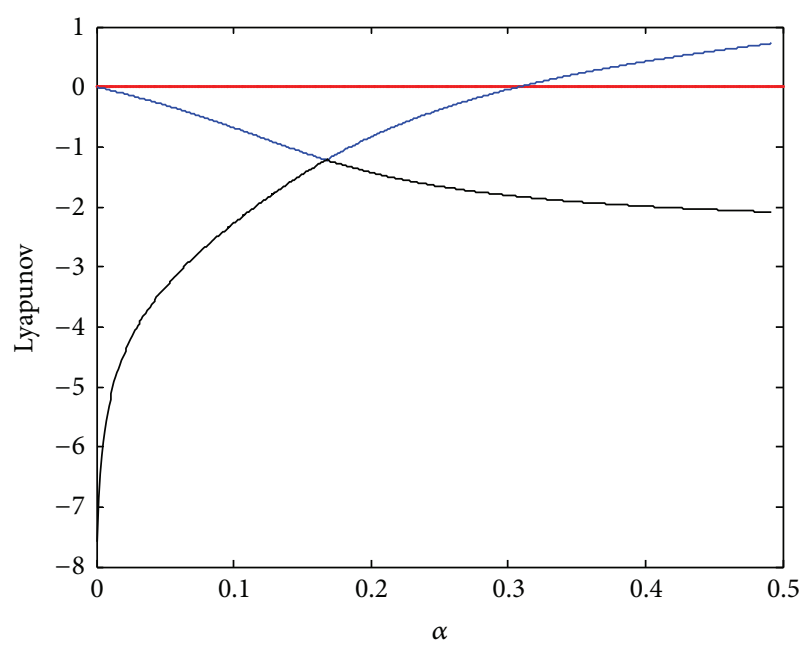

FIGURE 2: The related maximal Lyapunov exponents diagram as a function of the parameter $\alpha$.

Figure 1 shows the bifurcation diagram of the dynamical model with respect to parameter $\alpha$. As shown in Figure 1, the bifurcation diagram of the system (13) converges on the Nash equilibrium point $\left(q_{1}^{*}, q_{2}^{*}\right)=(2.030,2.122)$ when the parameter $\alpha<0.3082$. Period-doubling bifurcations appear at the parameter $\alpha=0.3082$. With the parameter $\alpha$ increasing continuously, point $E^{*}$ will be unstable, and chaotic behaviors will occur after a cascade of period-doubling bifurcation.

To analyze the parameter sets for which a periodic behavior occurs, we can study the maximal Lyapunov exponent depending on parameter $\alpha$. The chaos phenomenon will emerge when the maximal Lyapunov exponent is positive. Moreover, by comparing the bifurcation diagram in parameter $\alpha$, we can obtain a better understanding of the particular properties of the system. Figure 2 shows the related maximal Lyapunov exponents as a function of the parameter $\alpha$. From Figure 2, we can easily obtain the degree of the local stability

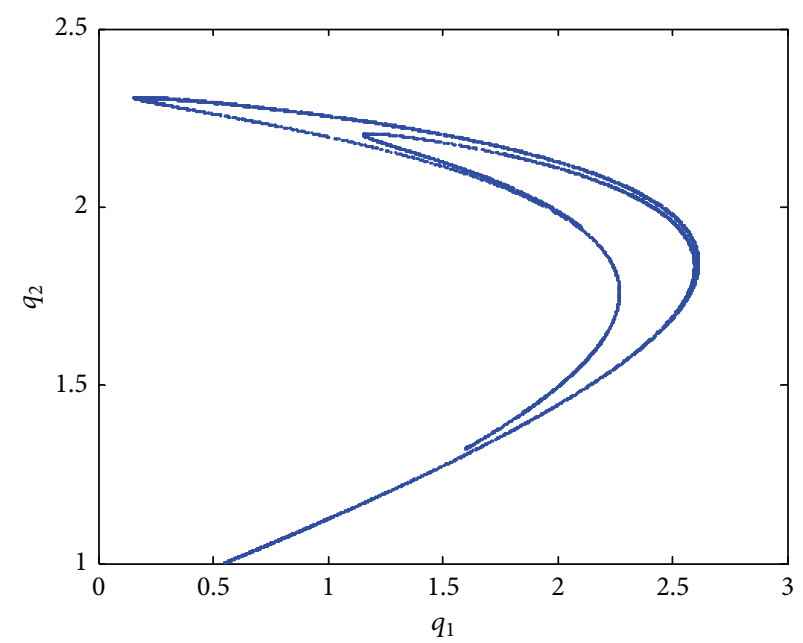

FIGURE 3: The strange attractor of the system at the parameter $\alpha=$ 0.46 .

for different values of the parameter $\alpha<0.3082$ and the parameter sets for which the system (13) converges to cyclic, aperiodic, and chaotic behavior [17].

Figure 3 represents the graph of a strange attractor of the system (13) for the values of parameter $\alpha=0.46$, which exhibits a fractal structure. The typical characteristic of the strange attractor can be described by the fractal dimension. We examine the important characteristic of neighboring chaotic orbits to see how rapidly they separate from each other [1-5]. In our case, the two-dimensional map has the Lyapunov dimension defined as follows:

$$
d_{L}=1+\frac{\lambda_{1}}{\left|\lambda_{2}\right|},
$$

where $\lambda_{1}$ and $\lambda_{2}$ are the Lyapunov exponents.

Two Lyapunov exponents exist and are $\lambda_{1}=0.1277$ and $\lambda_{2}=-1.873$ at $\alpha=0.46$; thus, the chaotic attractor presented in Figure 3 has fractal dimension $d_{L}=1+0.068=1.068$, which exhibits a fractal structure and has the same fractal dimension as the Henon map [16].

The sensitive dependence on initial conditions is one of the features of chaotic behavior of the system. To demonstrate the sensitivity to initial conditions of the system (13), two trajectories with different initial values $\left(q_{1_{0}}, q_{2_{0}}\right)=(0.3,0.4)$ and $\left(q_{1_{0}}+0.00001, q_{2_{0}}\right)=(0.30001,0.4)$ are computed for variables $q_{1}$ and $q_{2}$. The results are presented in Figure 4 plotted against the time $t$ with the parameter $\alpha=0.46$. In Figure 4 , at the beginning the time series, $q_{1}$ and $q_{2}$, are depicted simultaneously for various initial values and are indistinguishable in all cases considered. After approximate 30 iterations, the differences between them are built up rapidly. It follows that very little difference between initial values will have a great impact on results of the game, and thus the system (13) presents the typical characteristics of chaos behavior.

By means of numerical analysis and simulation, it is shown above that the game with heterogeneous operators is a dynamic process. The bounded rational operator is in 


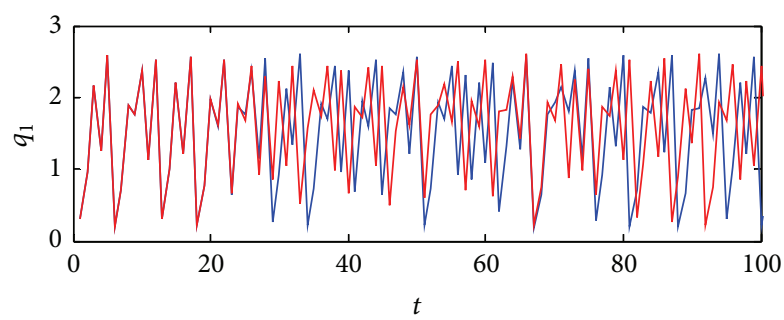

(a)

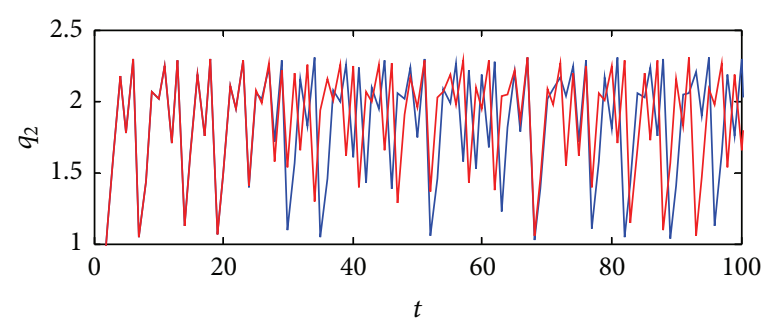

(b)

FIGURE 4: The sensitive dependence on initial conditions; (a) the two orbits of $q_{1}$-coordinates and (b) the two orbits of the $q_{2}$-coordinates for system $(13)$ at $\left(q_{1_{0}}, q_{2_{0}}\right)=(0.3,0.4)$ and the parameter $\alpha=0.46$.

the dominant position in the process of the game and controls the whole process by changing the adjustment speed $\alpha$ of the production. And the naive operator in the passive position is only adjusted on the basis of the production of the bounded rational operator.

The two operators gradually tend to Nash equilibrium in the process of a dynamic repeated game when the adjustment speed is in $\alpha<0.3082$. Then, the fixed broadband market is in a relatively stable competition environment. According to the characteristics of the fixed broadband market, users are in a rising state, which causes the operators to conduct a new round of the dynamic repeated game once again until reaching the Nash equilibrium of the system. However, when the adjustment speed $\alpha>0.3082$, the Nash equilibrium of the system will enter the unstable region because the adjustment speed $\alpha$ is too high, and the passive operator also becomes unstable. The complex dynamic phenomena such as period-doubling bifurcation and chaos will appear in the fixed broadband market. With the fixed broadband market in the state of disordered competition, operators will not be able to gain stable profits, and the market appears unpredictable. In these chaotic conditions, because the operators' decisionmaking levels and the cost are different, small changes in the initial production will have a great influence on the final results as the market becoming more unpredictable.

\section{Chaos Control in the Fixed Broadband Market}

When the chaotic phenomenon appears in the fixed broadband market, both operators are reluctant to face it, and thus they will take certain measures to delay or eliminate the occurrence of chaos and put the fixed broadband market in the stable state. This paper adopts the delay feedback control method put forward by Pyragas [18] to control the chaos of the system. In Pyragas' method, the control input is based on the difference between the $T$-time delayed state and the current state [17]. The feedback control input is

$$
u(t)=k(x(t+1-T)-x(t+1)), \quad t>T,
$$

where $T$ is the delayed time and $k$ is the controlling factor.

The bounded rational operator is in the dominant position in the fixed broadband market and implements the strategy of chaos control. By choosing $T=1$, the controlled system takes the form

$$
\begin{aligned}
& q_{1}(t+1) \\
& =q_{1}(t)+\alpha q_{1}(t) \\
& \quad \times\left(\left(a-\frac{d_{1}}{1+r_{12} q_{2}}\right)-2\left(b+\frac{c_{1}}{1+r_{12} q_{2}}\right) q_{1}(t)-b q_{2}(t)\right) \\
& \quad+k\left(q_{1}(t)-q_{1}(t+1)\right) \\
& q_{2}(t+1)=\frac{1}{2\left(b+\left(c_{2} /\left(1+r_{21} q_{1}\right)\right)\right)} \\
& \quad \times\left(\left(a-\frac{d_{2}}{1+r_{21} q_{1}}\right)-b q_{1}(t)\right) .
\end{aligned}
$$

The Jacobian matrix at Nash equilibrium point $E^{*}=\left(q_{1}^{*}, q_{2}^{*}\right)$ takes the form

$$
J\left(E^{*}\right)=\left[\begin{array}{cc}
1+\frac{\alpha}{(k+1)}\left(8-\frac{2}{1+(1 / 2) q_{2}^{*}}-\left(2+\frac{8}{1+(1 / 2) q_{2}^{*}}\right) q_{1}^{*}-\frac{1}{2} q_{2}^{*}\right) & \frac{\alpha}{(k+1)} q_{1}^{*}\left(\frac{1+2 q_{1}^{*}}{\left(1+(1 / 2) q_{2}^{*}\right)^{2}}-\frac{1}{2}\right) \\
\frac{\left(2 /\left(1+q_{1}^{*}\right)^{2}\right)-(1 / 2)}{1+\left(6 /\left(1+q_{1}^{*}\right)\right)}+3 \frac{16-\left(4 /\left(1+q_{1}^{*}\right)\right)-q_{1}^{*}}{\left(1+\left(6 /\left(1+q_{1}^{*}\right)\right)\right)^{2}\left(1+q_{1}^{*}\right)^{2}} & 0
\end{array}\right] .
$$

According to the necessary and sufficient condition for the local stability of the Nash equilibrium (11), we can obtain that system (16) is stable when $\alpha<0.3082(k+1)$ and other parameter values are fixed. The value region of parameter $\alpha$ in controlling factor $k=0$ and in the original system is the same, which leads to controlled system degradation for the original system. When the controlling factor $k=0.2$, the stable region of the Nash equilibrium for the controlled system becomes 


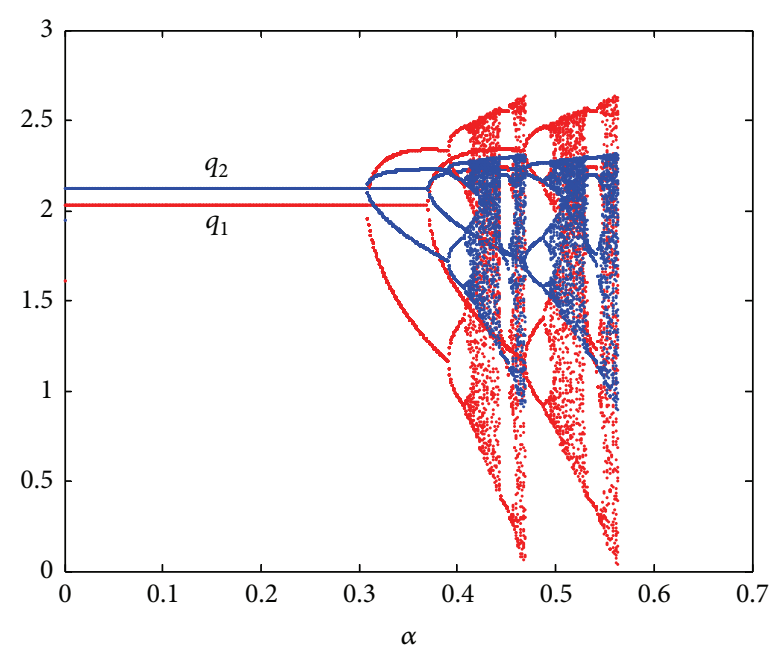

FIGURE 5: The bifurcation diagram before and after the system is controlled with the controlling factor $k=0.2$.

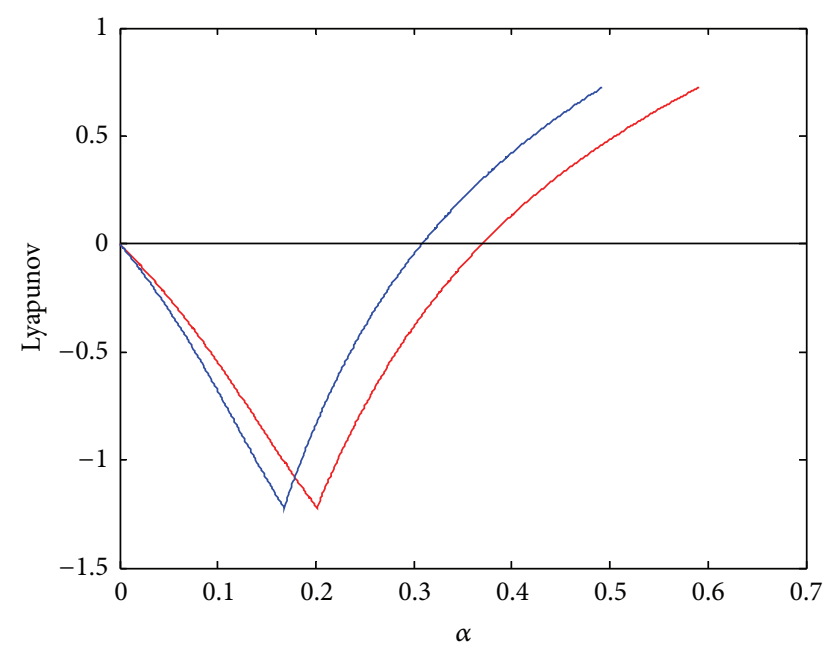

FIgURE 6: The maximal Lyapunov exponents diagram before and after the system is controlled with the controlling factor $k=0.2$.

larger. The value region of adjustment speed $\alpha$ increases from $\alpha=[0,0.3082]$ before being controlled to $\alpha=[0,0.3698]$ after being controlled, which is shown in Figures 5 and 6.

As the controlling factors further increase, the chaotic state of the system is controlled until it is fully eliminated gradually. Because the system is stable with $\alpha<0.3082(k+$ $1)$, we can obtain that system (16) is stable when $k>$ $(\alpha / 0.3082)-1$. The evolution of the system as controlling factor $k$ is varied as shown in Figure 7 and the corresponding maximum Lyapunov exponents in Figure 8 show the stability of the controlled system for $\alpha=0.46$. According to the figures, we can draw that when the controlling factor $k>$ 0.4925 , the system is stable around the Nash equilibrium point $\left(q_{1}^{*}, q_{2}^{*}\right)=(2.030,2.122)$. As shown in Figure 9 , after inserting controlling factor $k=0.3$, a game of approximately

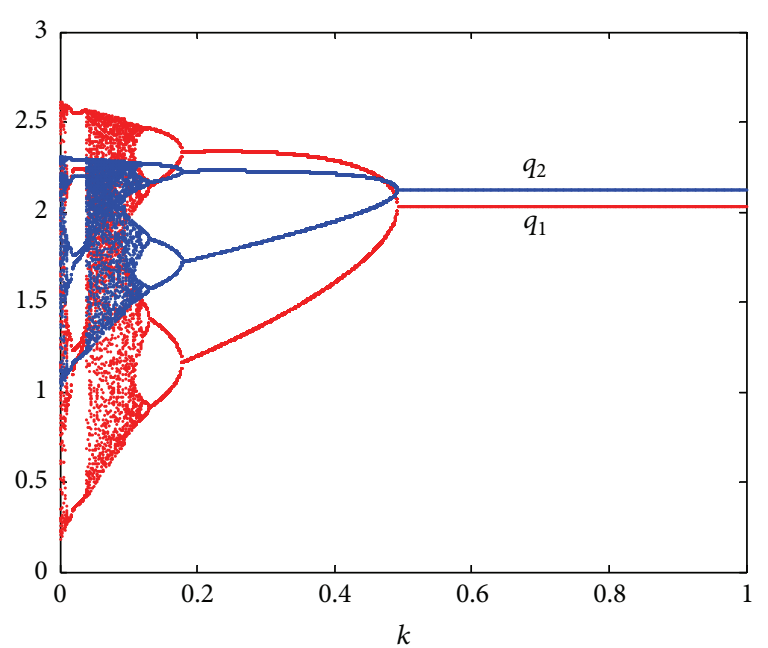

FIGURE 7: The bifurcation diagram with respect to the controlling factor $k$ with the parameter $\alpha=0.46$.

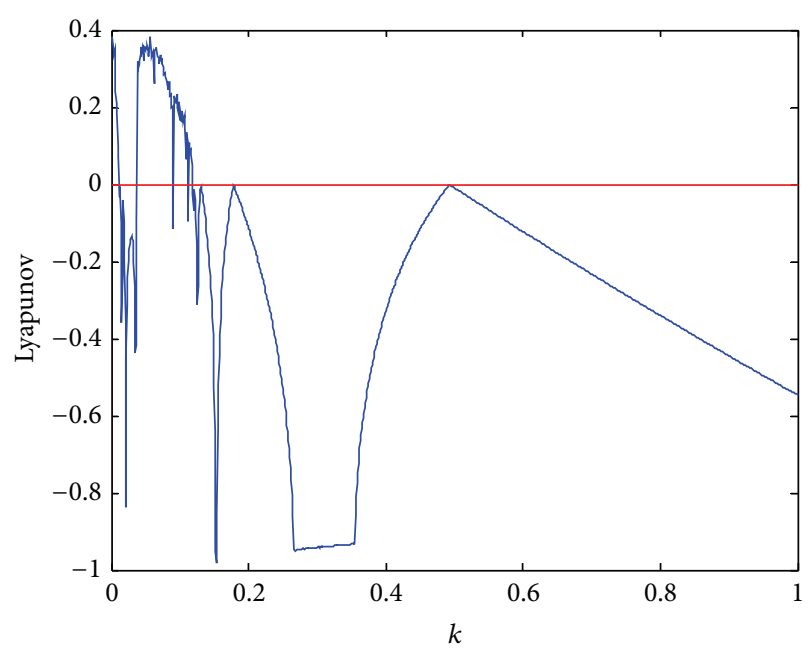

FIGURE 8: The related maximal Lyapunov exponents as a function of the controlling factor $k$ with the parameter $\alpha=0.46$.

30 times would lead to the stability of the controlled system at the 2-period points $(1.381,2.331)$ and $(1.838,2.226)$. As shown in Figure 10, after inserting controlling factor $k=0.8$, a game of approximately 20 times can lead to the stability of the controlled system at the Nash equilibrium point $\left(q_{1}^{*}, q_{2}^{*}\right)=$ $(2.030,2.122)$.

For $0<\alpha<1$ and the Nash equilibrium stability condition $\alpha<0.3082(k+1)$, we can obtain that the Nash equilibrium point in the entire value region $\alpha=[0,1]$ is stable when the controlling factor $k>2.2446$. As shown in Figure 11, when the controlling factor $k=2.5$ and the parameter $\alpha=[0,1]$, the controlled system is stable at the Nash equilibrium point $\left(q_{1}^{*}, q_{2}^{*}\right)=(2.030,2.122)$. According to the analysis above, the insertion of controlling factor $k$ delays or eliminates bifurcation in the controlled system compared 


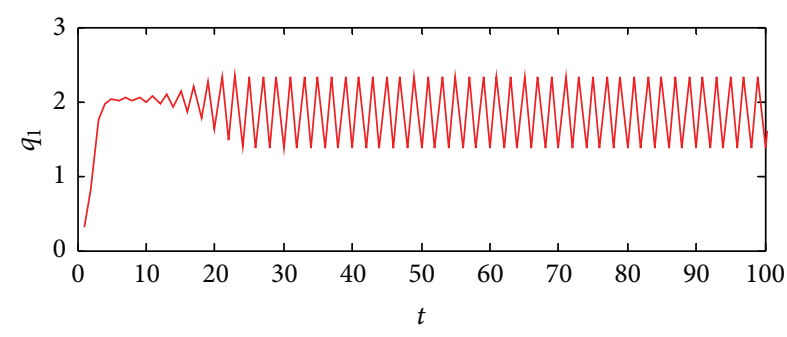

(a)

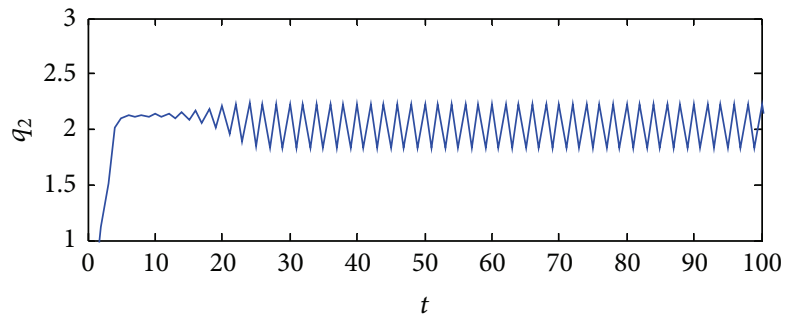

(b)

FIGURE 9: The 2-period status of the system as a function of time $t$ with the controlling factor $k=0.3$ and the parameter $\alpha=0.46$.

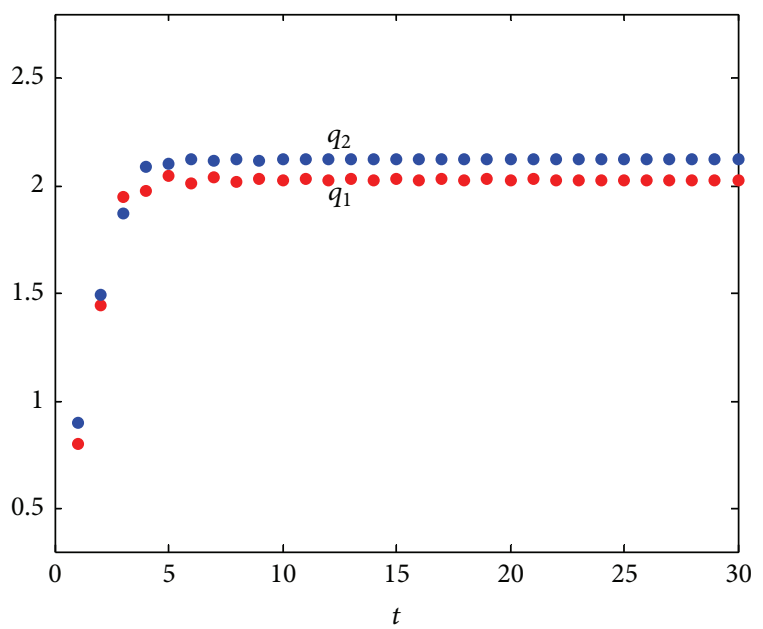

FIgURE 10: The stable status of the system as a function of time $t$ with the controlling factor $k=0.8$ and the parameter $\alpha=0.46$.

with the original system, which delays or prevents the appearance of chaos.

The dominant operator can control the chaotic status in the fixed broadband market by the delaying feedback control method. By adjusting the delay feedback controlling factor $k$, the fixed broadband market can be placed in a stable state. With the increase of factor $k$, the chaos in fixed broadband market gradually comes under control and is completely eliminated, which is good for all operators in the Chinese fixed broadband market.

\section{Conclusions}

Based on the differences of the development of the operators, this paper sets up one dynamic game model with heterogeneous operators. By combining the relevant theories of system dynamics, the dynamic evolution process of the model has been qualitatively analyzed. When the dominant operator adjusts the speed parameter $\alpha$ in the stable region, the production gradually tends to the Nash equilibrium after repeated games, and the fixed broadband market is in a stable state of benign competition. When parameter $\alpha$ is beyond

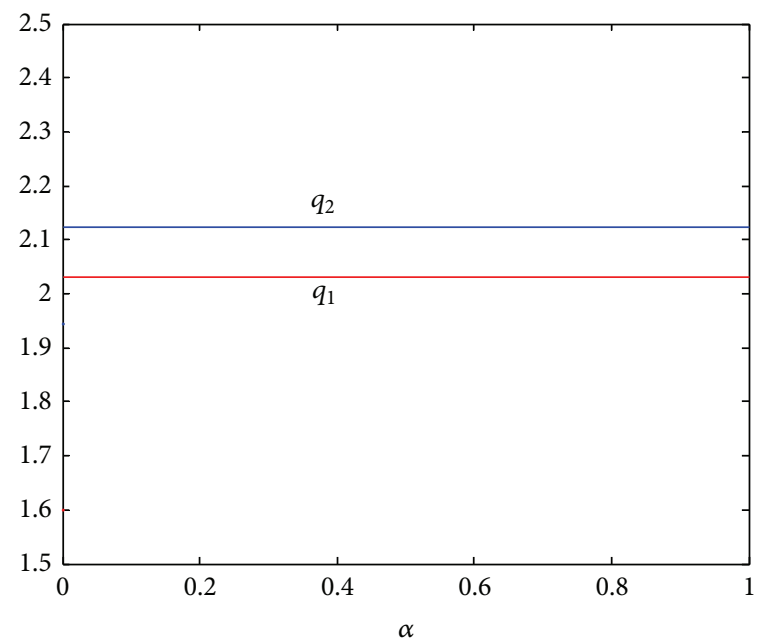

FIgUre 11: The stable status of the system as a function of the parameter $\alpha$ with the controlling factor $k=2.5$.

the stable region, there appear periodic bifurcation and chaos in the fixed broadband market. When chaos occurs, it will do harm to each of the competitors and the fixed broadband market will become abnormal, irregular, and unpredictable.

To solve this problem, this paper uses the delay feedback control method to solve the chaotic status in the fixed broadband market, which makes duopoly quantity stabilize on the Nash equilibrium point. The results show that the stable region of the Nash equilibrium increases after applying the delay feedback control, and thus chaotic state in the fixed broadband market will be postponed or even eliminated, and Chinese fixed broadband market is in a stable state of oligopoly competition. The research results have an important theoretical and practical significance to Chinese fixed broadband market. This paper shows guidance for Chinese fixed broadband companies to formulate quantity strategies and is helpful for the government to formulate relevant policies to issue more licenses.

\section{Conflict of Interests}

The authors declare that there is no conflict of interests regarding the publication of this paper. 


\section{Acknowledgment}

The authors are grateful to Management School, Jilin University, for supporting this work.

\section{References}

[1] H. N. Agiza and A. A. Elsadany, "Chaotic dynamics in nonlinear duopoly game with heterogeneous players," Applied Mathematics and Computation, vol. 149, no. 3, pp. 843-860, 2004.

[2] J. Ma and X. Pu, "Complex dynamics in nonlinear triopoly market with different expectations," Discrete Dynamics in Nature and Society, vol. 2011, Article ID 902014, 12 pages, 2011.

[3] A. A. Elsadany, "Competition analysis of a triopoly game with bounded rationality," Chaos, Solitons \& Fractals, vol. 45, pp. 1343-1348, 2012.

[4] H. Wang and J. Ma, "Complexity analysis of a CournotBertrand duopoly game model with limited information," Discrete Dynamics in Nature and Society, vol. 2013, Article ID 287371, 6 pages, 2013.

[5] B. Xin and Y. Li, "Bifurcation and chaos in a price game of irrigation water in a coastal irrigation district," Discrete Dynamics in Nature and Society, vol. 2013, Article ID 408904, 10 pages, 2013.

[6] A. Matsumoto and F. Szidarovszky, "Nonlinear delay monopoly with bounded rationality," Chaos, Solitons \& Fractals, vol. 45, no. 4, pp. 507-519, 2012.

[7] W. Z. Ji, "Chaos and control of game model based on heterogeneous expectations in electric power triopoly," Discrete Dynamics in Nature and Society, vol. 2009, Article ID 469564, 8 pages, 2009.

[8] J.-G. Du, T. Huang, and Z. Sheng, "Analysis of decision-making in economic chaos control," Nonlinear Analysis: Real World Applications, vol. 10, no. 4, pp. 2493-2501, 2009.

[9] L. Chen and G. Chen, "Controlling chaos in an economic model," Physica A, vol. 374, no. 1, pp. 349-358, 2007.

[10] H. N. Agiza, "On the analysis of stability, bifurcation, chaos and chaos control of Kopel map," Chaos, Solitons \& Fractals, vol. 10, no. 11, pp. 1909-1916, 1999.

[11] H. X. Yao, C. Y. Wu, D. P. Jiang, and J. Ding, "Chaos control in an investment model with straight-line stabilization method," Nonlinear Analysis: Real World Applications, vol. 9, no. 2, pp. 651-662, 2008.

[12] J. Du, T. Huang, Z. Sheng, and H. Zhang, "A new method to control chaos in an economic system," Applied Mathematics and Computation, vol. 217, no. 6, pp. 2370-2380, 2010.

[13] J. A. Holyst and K. Urbanowicz, "Chaos control in economical model by time-delayed feedback method," Physica A: Statistical Mechanics and its Applications, vol. 287, no. 3-4, pp. 587-598, 2000.

[14] F. Chen, J. H. Ma, and X. Q. Chen, "The study of dynamic process of the triopoly games in chinese $3 \mathrm{G}$ telecommunication market," Chaos, Solitons \& Fractals, vol. 42, no. 3, pp. 1542-1551, 2009.

[15] G.-I. Bischi and F. Lamantia, "Nonlinear duopoly games with positive cost externalities due to spillover effects," Chaos, Solitons \& Fractals, vol. 13, no. 4, pp. 701-721, 2002.

[16] E. M. Elabbasy, H. N. Agiza, A. A. Elsadany, and H. El-Metwally, "The dynamics of triopoly game with heterogeneous players," International Journal of Nonlinear Science, vol. 3, no. 2, pp. 8390, 2007.
[17] E. M. Elabbasy, H. N. Agiza, and A. A. Elsadany, "Analysis of nonlinear triopoly game with heterogeneous players," Computers \& Mathematics with Applications, vol. 57, no. 3, pp. 488-499, 2009.

[18] K. Pyragas, "Continuous control of chaos by self-controlling feedback," Physics Letters A, vol. 170, no. 6, pp. 421-428, 1992. 


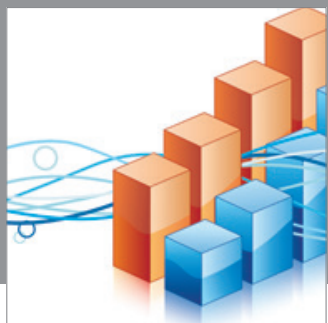

Advances in

Operations Research

mansans

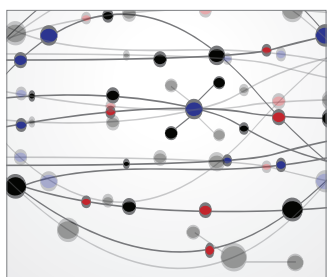

The Scientific World Journal
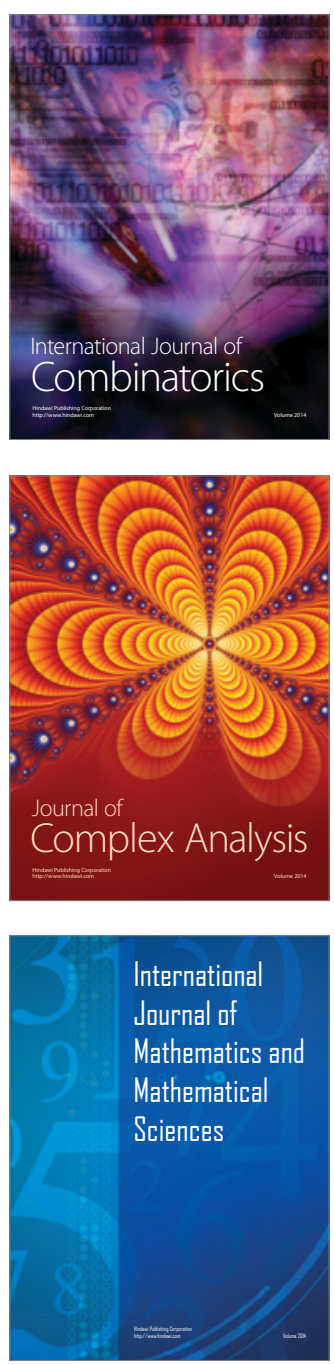
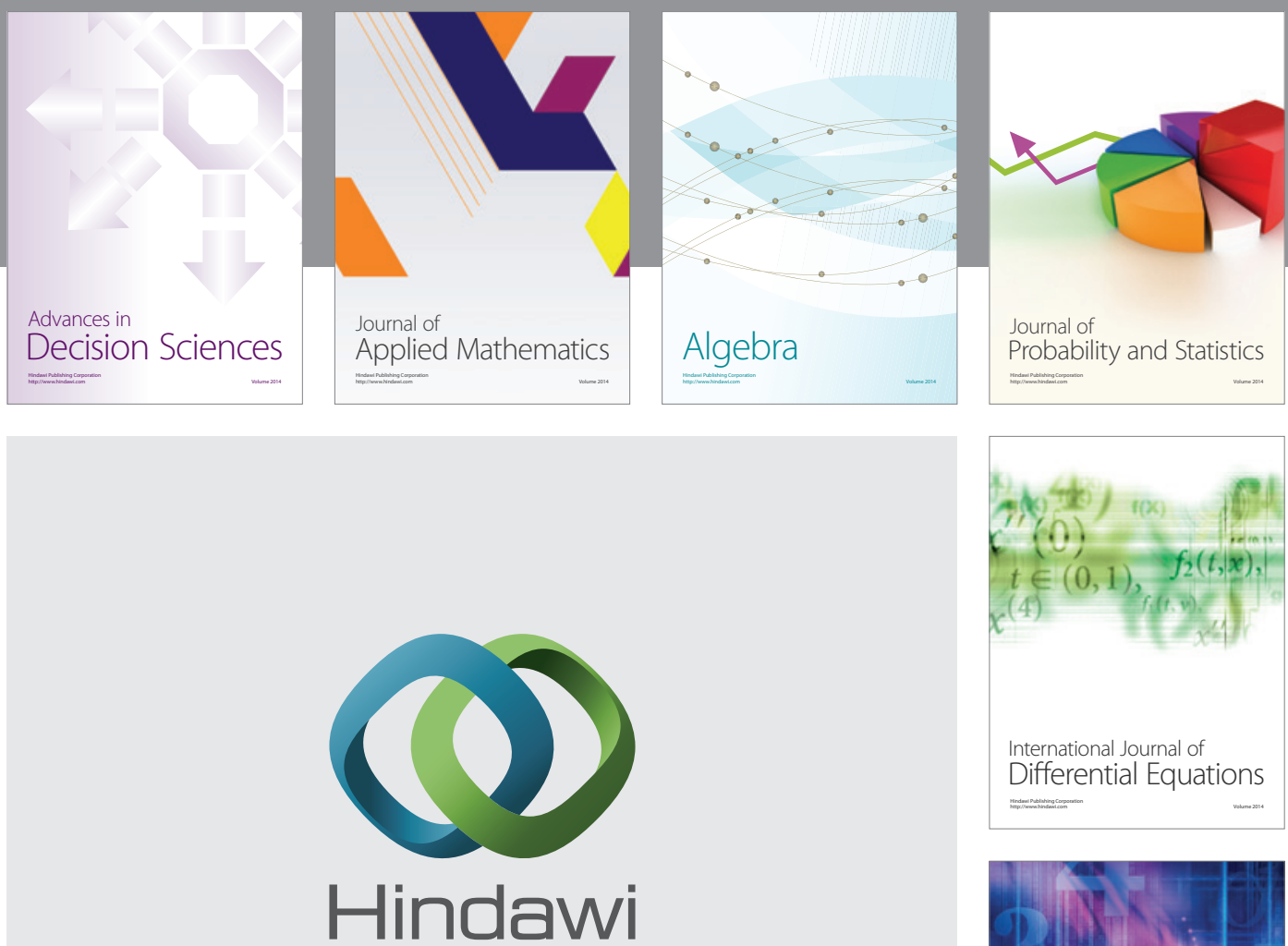

Submit your manuscripts at http://www.hindawi.com
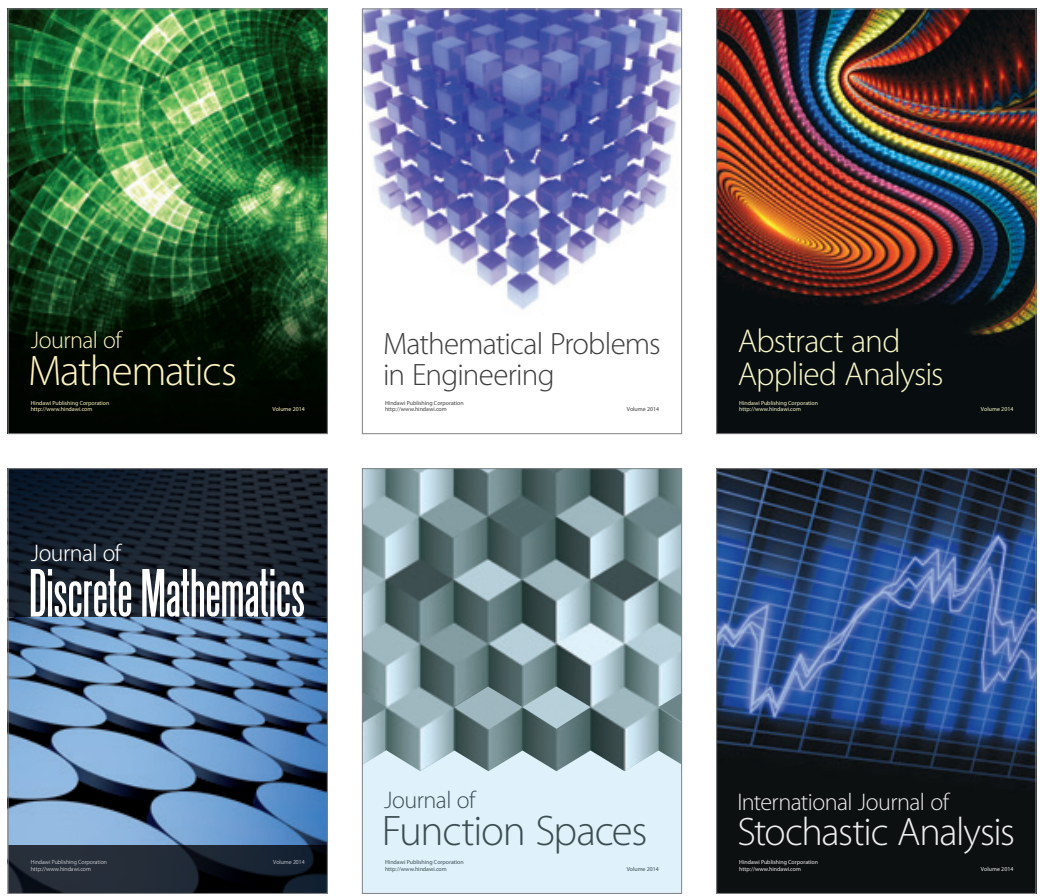

Journal of

Function Spaces

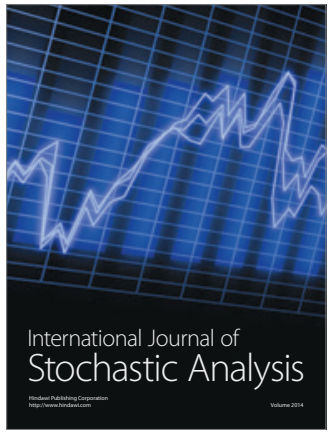

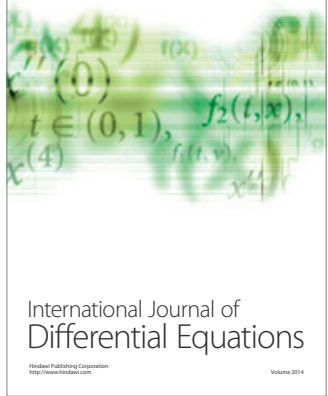
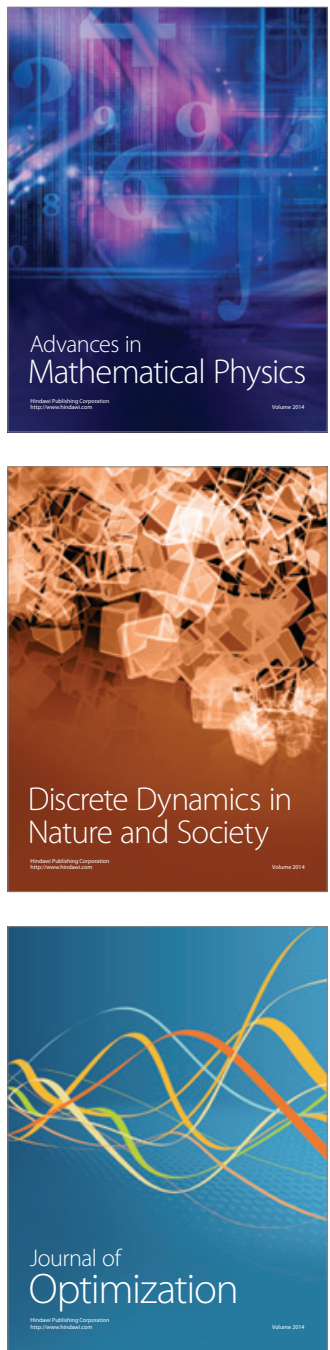